\title{
Poesía eres tú: innovación docente para la enseñanza de la lírica de la segunda mitad del siglo XIX
}

\section{Poetry is you: innovation in the teaching of lyric of the second half of the XIX century}

EMILIO José OCAMPos Palomar

ORCID: http://orcid.org/0000-0002-2663-7718

Universidad de Sevilla

Departamento de Literatura

Española e Hispanoamericana

eocampos@us.es

Fecha de recepción:

Fecha de aceptación:

DOI: http://dx.doi.org/10.12795/9788447221912.065

Pp.: 1500-1518 
Resumen

Este trabajo tiene como objetivo la exposición del desarrollo de un Ciclo de Mejora en el Aula (CIMA) aplicado a la asignatura Literatura Española del siglo XIX del Grado en Filología Hispánica, durante el primer cuatrimestre del curso 2019-2020. Para la elaboración y el funcionamiento exitoso de dicho CIMA se ha tenido en cuenta un sistema didáctico organizado en tres ejes interrelacionados (contenidos, metodología y evaluación) y una serie de aprendizajes docentes como la descripción de la práctica de enseñanza, la reformulación del modelo metodológico, el diseño de secuencias de actividades acorde a ese modelo, la aplicación de contenidos de diferentes tipos y sus relaciones, el empleo de preguntas que atraigan el interés del estudiante, la producción de un cuestionario (inicial y final), así como de escaleras de aprendizaje, y la organización de la práctica docente en torno a unos principios didácticos.

Palabras clave: Docencia universitaria, innovación docente, Poesía, Siglo XIX, Filología.

\section{Abstract}

The aim of this work is to expose the development of a Ciclo de Mejora en el Aula (CIMA-Improvement Cycles in Classroom-ICIC) applied to the subject of Spanish XIX Century Literature in the Bachelor's Degree of Hispanic Philology, in the first semester 2019-2020. For the purpose of said $\mathrm{CICC}$, it has been taken into account a didactic system organized in three interrelated core ideas (content, methodology and assessment) and a series of teaching techniques such as teaching practice description, reformulation of a methodological model, the sequencing design of the activities, the application of contents, eliciting to attract students attention, the production of a questionnaire (initial and final), learning steps and the organization of the teaching practice regarding didactic principles.

Key words: University teaching, teaching innovation, poetry, XIX century, Philology.

Jornadas de Formación e Innovación Docente del Profesorado | № 2 (2019) Esta obra se distribuye con la licencia Creative Commons Reconocimiento-NoComercial-SinObraDerivada 


\section{Breve descripción del contexto}

Mi Ciclo de Mejora en el Aula lo he proyectado en la asignatura Literatura Española del siglo XIX del Grado en Filología Hispánica. Es una materia obligatoria del tercer curso del Grado y acoge a una gran cantidad de alumnos, por lo que se encuentra dividida en tres grupos. Mi encargo y responsabilidad docente en la materia se desarrolla en el grupo 3, junto a otra profesora del Departamento de Literatura Española e Hispanoamericana con amplio recorrido en el ámbito investigador y en la docencia universitaria. Concretamente, me ocupo de las clases teórico-prácticas sobre la lírica en la segunda mitad del siglo XIX.

El grupo, de 66 alumnos matriculados (suelen asistir en torno a unos 45 alumnos), es muy heterogéneo en cuanto a edades y nacionalidades, lo que lleva a una dificultad en la cohesión del aprendizaje. Aun así, me siento muy cómodo con la docencia de dicha asignatura, debido a que los contenidos se mueven en los márgenes de mi tesis doctoral y en mis intereses como investigador. Además, es la segunda vez que tengo el privilegio de impartirla, disponiendo ya de un recorrido y experiencia docente en la enseñanza de la Literatura Española del siglo XIX.

\section{Diseño previo del CIMA}

\section{Mapa de contenidos y problemas}

El mapa de contenidos y problemas está diseñado para integrar en la docencia diferentes tipos de contenidos que comprenden datos, procedimientos intelectuales, conceptos y valores. Se ha buscado, por un lado, la interrelación de los contenidos para lograr una mayor cohesión del tema y, por otro, la organización en contenidos 
estructurales e imprescindibles y contenidos derivados de los mismos. Asimismo, el mapa presenta una serie de problemas formulados a través de preguntas atrayentes que ayudan a trabajar los contenidos. Esas preguntas constituyen el cuestionario inicial y final con el que he elaborado las escaleras de aprendizaje para la evaluación colectiva e individual.

Por otro lado, con este mapa de contenidos pretendo reivindicar la figura de las poetas del siglo XIX y cuestionar el olvido y abandono de las mismas en una Historia Literaria construida por una crítica masculina. De esta forma, el grueso y el centro del mapa lo ocupa Rosalía de Castro y la revisión feminista, dando así importancia a los contenidos actitudinales.

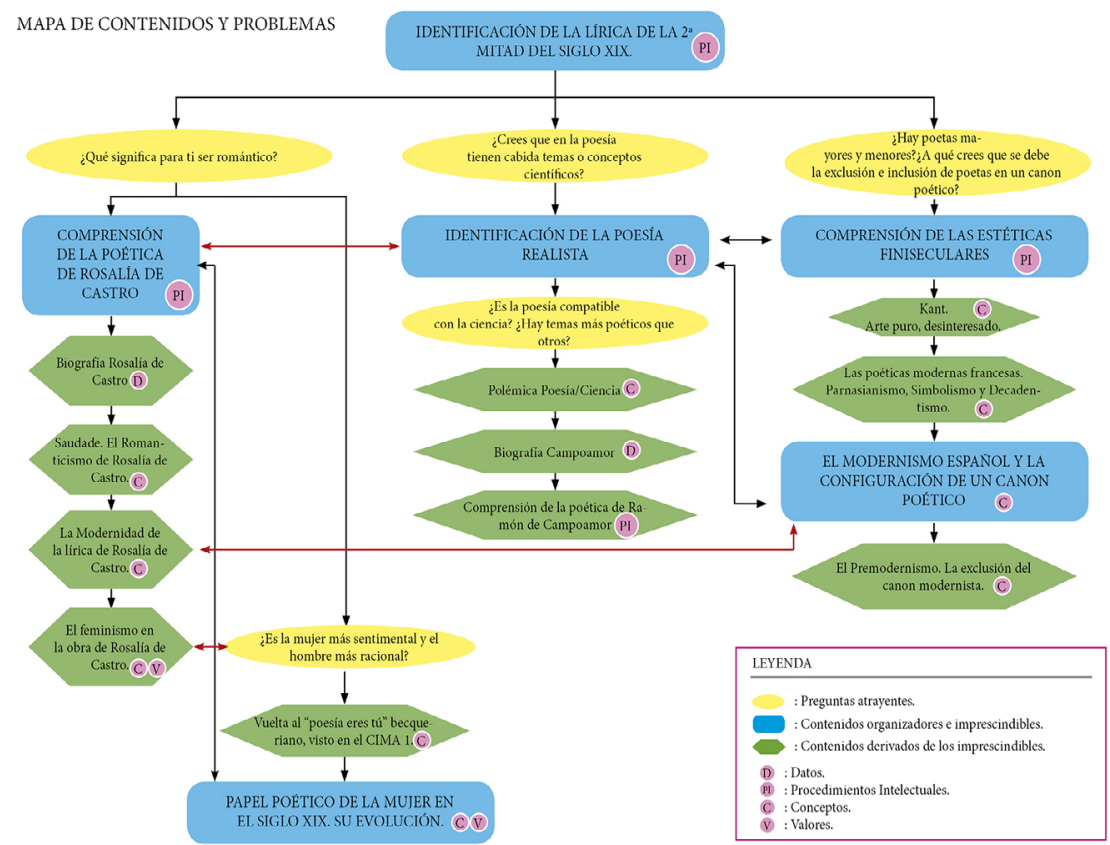

Figura 1. Mapa de contenidos y problemas

Jornadas de Formación e Innovación Docente del Profesorado | № 2 (2019) Esta obra se distribuye con la licencia Creative Commons Reconocimiento-NoComercial-SinObraDerivada 


\section{Modelo metodológico posible y secuencia de actividades}

Tras la identificación de mi "modelo metodológico real" y la superación del mismo a raíz de la elaboración de un "modelo metodológico ideal", he diseñado un "modelo metodológico posible" que tiene en cuenta las dificultades de aplicación del ideal, pero que no lo pierde de vista. Es decir, he aplicado un modelo que he sido capaz de llevar a cabo, teniendo siempre como referente el modelo ideal (Alba y Porlán, 2017: 42).

El modelo se ha construido para que empiece y termine en una sesión de 2 horas. Así, y de manera cíclica, tiene lugar durante las 4 sesiones de duración del CIMA. En cada sesión se parte de un problema que lleva a los alumnos a unas ideas iniciales, seguidamente se desarrolla una fase de contraste (con actividades que combinan la teoría y la práctica) que logra el replanteamiento de las ideas previas de los alumnos hacia otras nuevas y, finalmente, se cierra la sesión con una sintesis que reagrupe esas ideas y sirva de conclusión. De esta forma, se atiende a puntos clave expresados por Bain como el hecho de partir de un problema o pregunta intrigante (2004: 115) o que el profesor sirva de guía, ayude a sus estudiantes a pensar, a que aprendan por sí mismos (2004: 130). Un modelo, en definitiva, que se basa en lo que Bain denomina "educación centrada en el estudiante y no educación centrada en la disciplina o en el profesor" (Bain, 2004: 128).

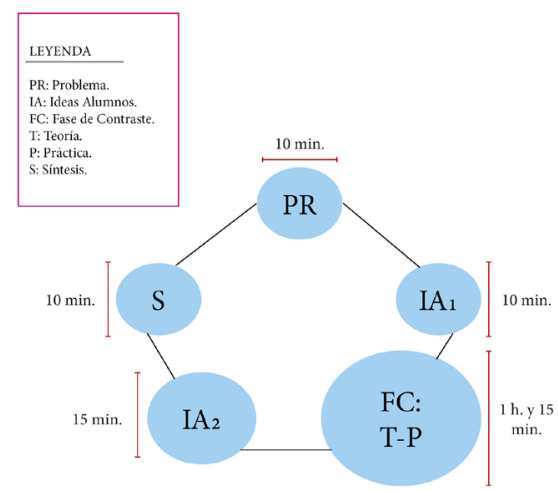

Figura 2. Modelo metodológico posible

Jornadas de Formación e Innovación Docente del Profesorado | № 2 (2019) Esta obra se distribuye con la licencia Creative Commons 
A continuación detallo las actividades realizadas para las 4 sesiones en las que se distribuye el CIMA:

\title{
Tabla 1. Secuencia de actividades del ciclo de mejora
}

\author{
SESIÓN 1 \\ Título \\ Correspondencia Contenido \\ Tiempo \\ con el modelo \\ metodológico \\ Desarrollo de \\ PR e IA1 \\ - Preguntar a los estudiantes: ¿Qué \\ $20 \mathrm{~min}$ \\ una pregunta \\ significa para ti ser romántico? \\ atrayente $\mathrm{y}$ \\ respuesta de los \\ -Tiempo para respuestas e \\ intercambio de opiniones entre los \\ estudiantes a la \\ alumnos. \\ misma \\ Biografia Rosalía FC: T \\ -Exposición, a través del Power \\ $15 \mathrm{~min}$. \\ de Castro \\ Saudade \\ FC: T-P \\ Point, de los acontecimientos más \\ relevantes de la vida y obra de \\ Rosalía de Castro. \\ -Explicación teórica del concepto \\ $30 \mathrm{~min}$. \\ y comentario de los siguientes \\ poemas: poema XVIII de "Vaguedás" \\ (Follas novas, 1880), poema XX de \\ "Do íntimo" (Follas novas) y poema $V$ \\ de "As viúdas dos vivos e as viúdas \\ dos mortos" (Follas novas). Lectura \\ en gallego y en español de los \\ poemas. \\ La lírica $\quad$ FC: T-P
moderna \\ -Comentario y reflexión de textos \\ $30 \mathrm{~min}$ \\ críticos que consideran a Rosalía \\ precursora, junto a Bécquer, del \\ Simbolismo en España. \\ Ideas finales \\ IA2

\begin{abstract}
- Volver a realizar la pregunta “¿Qué significa para ti ser romántico?" a la espera de un cambio en las respuestas iniciales gracias a las actividades realizadas en la Fase de Contraste. Retomar conceptos teóricos para una puesta en común. -Tiempo para respuestas, reflexiones e intercambio de opiniones entre los alumnos.
\end{abstract}

Jornadas de Formación e Innovación Docente del Profesorado | № 2 (2019) 
Cierre y S

conclusiones

\section{SESIÓN 3}

Desarrollo de una PR e IA1 pregunta atrayente y respuesta de los estudiantes a la misma

El Realismo poético FC: T

\section{Confrontación FC: T-P Poesía/Ciencia}

Acercamiento a $\quad$ FC: T-P
Campoamor

Ideas finales IA2
-A raíz de las respuestas y reflexiones $\quad 10$ min. de los alumnos se cerrará el tema, resumiendo y recapitulando las ideas fundamentales, fortaleciendo los conceptos claves a través de sus respuestas, intervenciones y reflexiones.

- Preguntar a los estudiantes: ¿Es la poesía 20 min. compatible con la ciencia? ¿Hay temas más poéticos que otros?

-Tiempo para respuestas e intercambio de opiniones entre los alumnos.

-Presentación y explicación, a través del Power15 min. Point, de la pintura El origen del mundo (1866) de Gustave Courbet.

- Características de la poesía realista.

-Recordatorio del Realismo y del Naturalismo, visto en clases anteriores.

-Explicación, apoyada en textos críticos, sobre 30 min. la polémica Poesía/Ciencia.

-Comentario de una selección de poemas: por un lado, poemas que se manifiestan a favor de la ciencia y el progreso tecnológico y, por otro, poemas que ven en la ciencia la muerte de la poesía, del misterio.

-Exposición, a través del Power Point, de los 30 min. acontecimientos más relevantes de la vida y obra de Ramón de Campoamor.

- Lectura y comentario del poema "El tren expreso", de Campoamor.

-Volver a preguntar “¿Es la poesía compatible 15 min. con la ciencia? ¿Hay temas más poéticos que otros?" a la espera de un cambio en las respuestas iniciales gracias a las actividades realizadas en la Fase de Contraste. Retomar conceptos teóricos para una puesta en común. -Tiempo para respuestas, reflexiones e intercambio de opiniones entre los alumnos. 
Cierre y S

conclusiones

SESIÓN 4

Desarrollo de

una pregunta

atrayente y

Ideas finales
FC: T-P

IA2
-A raíz de las respuestas y reflexiones de

$10 \mathrm{~min}$.

los alumnos se cerrará el tema, resumiendo

y recapitulando las ideas fundamentales,

fortaleciendo los conceptos claves a través de

sus respuestas, intervenciones y reflexiones. respuesta

de los

estudiantes a

la misma

Estética de

Kant y la

transgresión

FC: $T$

de la moral

burguesa

Las estéticas

francesas

El

Modernismo FC: T-P

español

PR e IA1 a
-Preguntar a los estudiantes: ¿Hay poetas mayores y menores? ¿A qué crees que se debe la exclusión e inclusión de poetas en un canon poético?

20

min.

-Tiempo para respuestas e intercambio de opiniones entre los alumnos.

-Explicación de le Estética de Kant. Arte puro, desinteresado.

-Explicación de los conceptos "l'art pour l'art",

"épater les bourgeois".

15

$\min$

-Presentación, a través de Power Point, de

algunas obras de Félicen Rops.

-Explicación del Parnasianismo, Simbolismo y Decadentismo. Su introducción en España. 30 -Lecturas y comentario de poemas de estas min. estéticas.

- Lecturas de poemas de poetas llamados "premodernistas". Comparación con poemas de poetas "modernistas".

-Explicación del "mito" de Darío y de la construcción interesada de una Historia 30 min. literaria, remitiendo al artículo "Cómo se escribe una historia literaria: Rubén Darío y el modernismo en España", de Richard Cardwell.

- Volver a preguntar “ ¿Hay poetas mayores y menores? ¿A qué crees que se debe la exclusión e inclusión de poetas en un canon poético?" a la espera de un cambio en las respuestas iniciales gracias a las actividades realizadas en la Fase de Contraste. Retomar 15 $\min$. conceptos teóricos para una puesta en común. -Tiempo para respuestas, reflexiones e intercambio de opiniones entre los alumnos. 
Cierre y conclusiones
-A raíz de las respuestas y reflexiones de los alumnos se cerrará el tema, resumiendo y recapitulando las ideas fundamentales, fortaleciendo los conceptos claves a través de 10 min. sus respuestas, intervenciones y reflexiones.

\section{Cuestionario inicial-final}

El cuestionario se confeccionó para acceder a los modelos mentales de los estudiantes. Para ello tuve en cuenta que no se trataba de averiguar si conocían o recordaban respuestas académicas, sino de conocer su forma de pensar, su punto de vista sobre lo estudiado (Rivero y Porlán, 2017: 78). De ahí surgieron las siguientes preguntas abiertas, relacionadas con un problema atractivo y que diesen lugar a respuestas espontáneas (Rivero y Porlán, 2017: 79):

1. ¿Qué significa para ti ser romántico?

2. ¿Es la mujer más sentimental y el hombre más racional?

3. ¿Crees que en la poesía tienen cabida temas o conceptos científicos?

4. ¿Es la poesía compatible con la ciencia? ¿Hay temas más poéticos que otros?

5. ¿Hay poetas mayores y menores? ¿A qué crees que se debe la exclusión e inclusión de poetas en un canon poético?

\section{Aplicación del CIMA}

\section{Relato resumido de las sesiones}

En la primera sesión me dirijo a los alumnos a propósito del cuestionario, mostrando mi interés a sus respuestas y advirtiéndoles que durante cuatro sesiones trabajaremos en torno a las preguntas del cuestionario. Al 
poner en común las respuestas de la primera pregunta se origina un debate entre los estudiantes sobre la historicidad del término "romántico". Noto cómo los estudiantes se muestran muy atentos al Power Point y prestan especial atención a aspectos de la vida de Rosalía de Castro como su orfandad o el hecho de que no quisiera escribir más en gallego. Muy contento por despertar su interés me dispongo a explicar el concepto de "saudade" y pasamos a la lectura de los poemas: comentamos entre todos los elementos románticos en Rosalía de Castro. A continuación, expongo las teorías de algunos críticos que presentan a Rosalía como precursora del Simbolismo y veo cómo los estudiantes identifican y relacionan los elementos simbolistas con la poética becqueriana. Esto me hace ver cómo los estudiantes están comprendiendo que Rosalía y Bécquer representan la lírica de la Modernidad. Me detengo aquí y refuerzo esta idea. Finalmente, tras la formulación de la pregunta inicial, observo cómo los estudiantes más participativos responden relacionando Romanticismo con Modernidad.

En la segunda sesión les recuerdo la pregunta dos del cuestionario y sin que haga falta sacar a la luz los tipos de respuestas, los estudiantes comienzan a dar las suyas. En esta pregunta participan más estudiantes, hay mayor interés. Sin embargo, son pocos los estudiantes que han leído los textos para este día (razón: falta de tiempo, muchas lecturas de otras asignaturas y que tan solo han dispuesto de un día para leer los textos). Esto me hace reflexionar y preguntarme si debo o no dejar más tiempo a los alumnos para las lecturas. Pido a una alumna que aún no ha participado en clase y que ha leído el texto de "Las literatas" su opinión. Apruebo su comentario y me detengo en algunos aspectos claves del texto como el hecho de que Rosalía denuncie que existan lectores que crean que es su marido quien le escribe los poemas. Compruebo, con alegría, cómo consiguen identificar que esto se debe al papel privado, doméstico que se le asigna a la mujer frente al papel 
público que se le asigna al hombre. Seguidamente pasamos a leer los poemas. Apruebo el acertado comentario de una alumna que anota que Rosalía al dudar sobre si tiene "alma de mujer" está respondiendo al "poesía eres tú" de Bécquer, la felicito y añado que responde a toda la concepción patriarcal que relega a la mujer al sentimiento y no a la razón, a la poesía y no al poeta. Me aseguro de que el resto de los estudiantes lo han comprendido, me confirman que sí. Como siempre, hay algunos más habladores y otros menos, lo que me hace preguntar constantemente para ver si aquellos que intervienen menos no se están perdiendo. Agotado el tiempo, no puedo volver a formular la pregunta inicial, pero lo considero innecesario porque los estudiantes han vuelto a hacerse la pregunta en el comentario de los poemas a raíz del material trabajado en clase. La actividad de contraste ha salido con más éxito de lo que esperaba.

En la tercera sesión hay menos asistencia. Sabiendo que las alumnas más participativas están siguiendo muy bien las sesiones, prefiero dirigirme a dos alumnas Erasmus para decirles si recuerdan su respuesta a la tercera pregunta del cuestionario. Veo que su modelo mental está bajo en la "escalera de aprendizaje", pero, para mi sorpresa, tras la explicación de la polémica decimonónica en torno a la poesía y la ciencia y la lectura de poemas que apoyan el desarrollo científico y otros que no, una de las alumnas Eramus me comenta que ahora entiende que la poesía también puede enfrentarse a las cuestiones científicas y no por ello perder lirismo. Compruebo que, una vez más, la actividad de contraste ha dado resultado antes de lo esperado. Refuerzo su respuesta y añado algunos comentarios para enlazar con el comentario de "El tren expreso". Los alumnos quedan muy satisfechos con el poema, me dicen que les ha enriquecido mucho. Para mi asombro, han leído, comprendido y, sobre todo, les ha gustado Campoamor, un autor cada vez más olvidado en el canon poético decimonónico. "El tren expreso" me lleva hasta el final 
de la sesión. No hay tiempo para la formulación de la pregunta inicial, pero no importa ya que las ideas nuevas de los alumnos se han producido con anterioridad.

En la cuarta sesión, tras preguntar por la última cuestión que teníamos pendiente, se abre la exposición de ideas. Varios estudiantes traen conocimientos previos sobre el tema, estudiados en otra materia, así que les advierto que nos centraremos en la problemática del canon finisecular, algo que los estudiantes desconocen. Tras explicar las características del Parnasianismo, el Simbolismo y el Decadentismo, analizamos los poemas de autores españoles y les indico que me identifiquen las características. Consiguen reconocerlas, otras salen a la luz con mi ayuda. Debatimos sobre las etiquetas "premodernista" y "modernista". Los estudiantes, sabiendo hacia dónde va la secuencia, manifiestan que la exclusión se debe al canon. Desarrollo la tesis de Cardwell sobre el "mito" de Rubén Darío y la construcción interesada de una historia literaria. Llego al final de la sesión y, tras la formulación de la pregunta inicial, los estudiantes se muestran participativos e interesados en profundizar en la cuestión del "mito" literario.

En un análisis de las cuatro sesiones, hay que decir que funcionó muy bien el modelo metodológico en torno a las preguntas atractivas planteadas en cada sesión. La actividad de contraste resultó positiva para que el estudiante desarrollase con la ayuda del profesor unas ideas nuevas que le permitiesen argumentar un discurso más riguroso y académico al establecido a través de las ideas iniciales. Especialmente, estoy muy contento con la segunda y tercera sesión, ya que no tuve que esperar a volver a formular la pregunta inicial para que surgieran las nuevas ideas de los alumnos, sino que estas surgieron cuando estábamos trabajando en la actividad de contraste. Ocurrió antes de lo que me esperaba y eso me sorprendió mucho.

Asimismo, la organización de la secuencia de actividades funcionó favorablemente, salvo en contadas ocasiones 
en las que tuve que improvisar, no siguiendo el mismo orden que establecí en la secuencia y añadiendo cuestiones que no tenía previstas. En algunos casos, y a pesar de estar muy pendiente del tiempo, tampoco ocurrió como estaba escrito en el papel la distribución temporal: hubo cuestiones que se desarrollaron con más rapidez de lo habitual, impidiendo profundizar y resolver dudas.

\section{Evaluación del aprendizaje de los estudiantes}

Los alumnos y alumnas evaluadas en los dos cuestionarios fueron 42. 4 estudiantes no asistieron al cuestionario final, así que no se les pudo evaluar. Del mismo modo, no se les pudo pasar el cuestionario final a los alumnos que no hicieron el inicial.

En un estudio contrastivo (por medio de las "escaleras de aprendizaje y evaluación") de cada una de las preguntas de los dos cuestionarios (inicial y final) se observa cómo la mayoría de los alumnos incrementan su nivel, muy pocos se mantienen en el mismo y ninguno baja de nivel.

A continuación presento las "escaleras de aprendizaje y evaluación" de dos de las preguntas del cuestionario inicial y final, concretamente las preguntas 1 y 3.

Pregunta 1: ¿Qué significa para tí ser romántico?

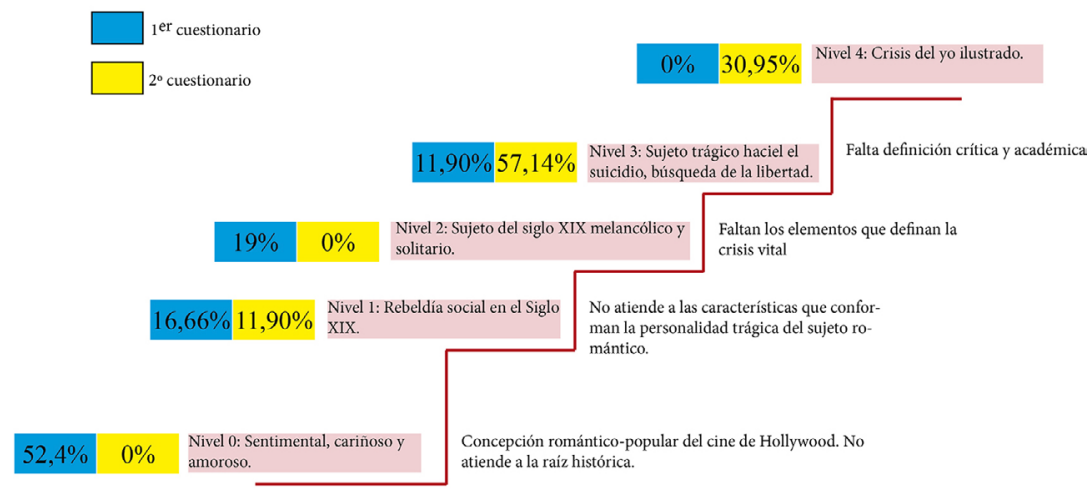

Figura 3. Escalera de aprendizaje de la primera pregunta

Jornadas de Formación e Innovación Docente del Profesorado | № 2 (2019) Esta obra se distribuye con la licencia Creative Commons Reconocimiento-NoComercial-SinObraDerivada 
En la pregunta número 1 (“¿Qué significa para ti ser romántico?") se observa un salto importante del nivel 0 al resto de niveles. Así queda superado el obstáculo "concepción romántico popular del cine de Hollywood. No atiende a la raíz histórica". De hecho, el porcentaje del nivel 0 en el primer cuestionario es 52,4\% y en el segundo cuestionario es $0 \%$. En el segundo cuestionario la mayoría de los alumnos se sitúan en el nivel 3: el 57,14\% frente al 11,9\% del primer cuestionario. Otro aspecto a destacar es la creación en el segundo cuestionario de un nuevo nivel: el nivel 4, en el que se sitúan el 30,95\% de estudiantes, superando el obstáculo "falta definición crítica y académica”.

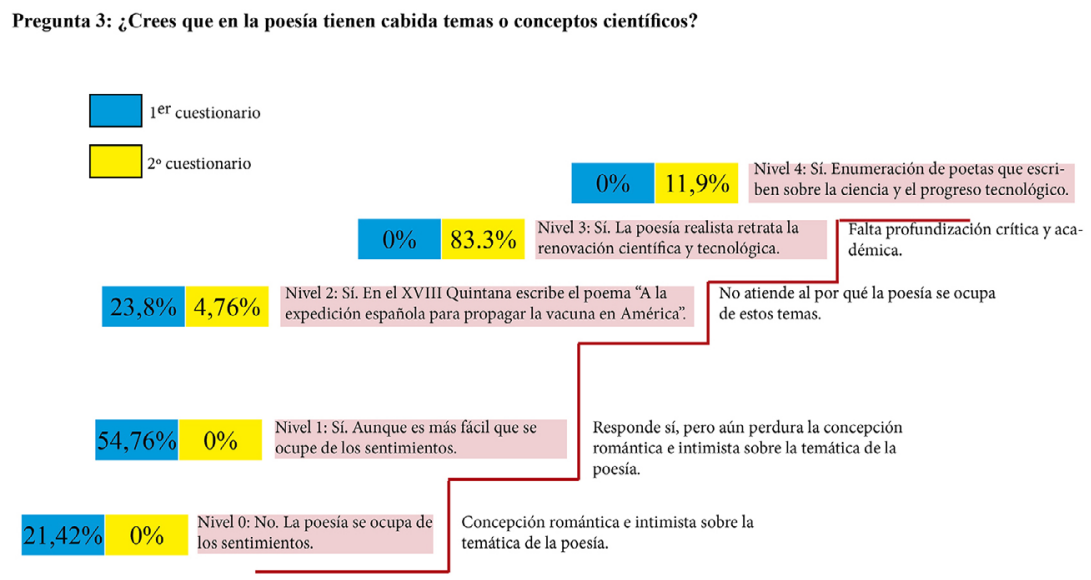

Figura 4. Escalera de aprendizaje de la tercera pregunta

En la pregunta número 3 (“¿Crees que en la poesía tienen cabida temas o conceptos científicos?") la mayor dificultad y, por tanto el mayor escalón, se sitúa entre el nivel 1 y el nivel 2. En el primer cuestionario un 21, 42\% de estudiantes se encuentra en el nivel 0 y un $54,76 \%$ en el nivel 1; sin embargo, en el segundo cuestionario este porcentaje queda a 0 y la mayor parte de los estudiantes (el 83, $3 \%)$ se encuentra en un nuevo nivel: el nivel 3, superando el obstáculo "responde sí, pero aún perdura la concepción romántica e intimista sobre la temática de la poesía". Hay que destacar en el segundo cuestionario la irrupción de dos niveles: por un lado, el ya mencionado nivel 3, donde 
están la mayoría de estudiantes; y, por otro, el nivel 4 con 11, $9 \%$ de estudiantes. A estos dos niveles llegan los que han conseguido superar el obstáculo "no atiene al por qué la poesía se ocupa de estos temas".

A continuación muestro una selección de 10 alumnos al azar para ver su evolución con respecto a las preguntas 1 y 3 :

Tabla 2. Cuadro de evaluación por estudiante NIVELES DE DESARROLLO INICIALES Y FINALES

PREGUNTA 1

PREGUNTA 3

Sujeto 1 Nivel $0 \quad$ Nivel $3 \quad \uparrow \quad$ Nivel 2 Nivel $3 \uparrow$

Sujeto 2 Nivel 0 Nivel $1 \quad \uparrow \quad$ Nivel 2 Nivel $2 \leftrightarrow$

Sujeto 3 Nivel 1 Nivel $1 \leftrightarrow \quad$ Nivel 0 Nivel2 $\uparrow$

Sujeto 4 Nivel 3 Nivel 4 个 Nivel 1 Nivel $3 \uparrow$

Sujeto 5 Nivel 2 Nivel $3 \quad \uparrow \quad$ Nivel 0 Nivel2 $\uparrow$

Sujeto 6 Nivel 3 Nivel 4 个 Nivel 2 Nivel 4 个

Sujeto 7 Nivel 2 Nivel $3 \uparrow \uparrow \quad$ Nivel 2 Nivel $4 \uparrow$

Sujeto 8 Nivel 2 Nivel $3 \quad \uparrow \quad$ Nivel $1 \quad$ Nivel $3 \uparrow$

Sujeto 9 Nivel 0 Nivel $3 \uparrow \quad$ Nivel 1 Nivel $3 \uparrow$

Sujeto 10 Nivel 1 Nivel $1 \leftrightarrow$ Nivel $1 \quad$ Nivel $3 \uparrow$

\section{Evaluación del CIMA}

La construcción y aplicación de un Ciclo de Mejora en el Aula me ha hecho cambiar como docente, reaprender mi profesión y, lo más importante, desarrollar unos principios didácticos con los que poder afrontar con compromiso las tareas de enseñanza y que expongo a continuación.

En cuanto al aprendizaje de alumnos y alumnas, considero fundamental centrar la enseñanza en los estudiantes, 
partir de sus ideas e indagar en sus modelos mentales; en definitiva, construir la docencia a raíz de sus ideas iniciales. He aprendido a que el profesor debe servir de guía en el aprendizaje de los alumnos. Uno de mis principios didácticos ha sido siempre interaccionar con los estudiantes, atenderlos, pero nunca había reflexionado sobre el hecho de partir de sus ideas iniciales. Esto ha sido posible gracias a la experiencia del CIMA y es algo que no voy a abandonar. Los alumnos me lo han agradecido respondiendo y siguiendo con atención las lecciones.

En cuanto a los contenidos de enseñanza, el mapa de contenidos y problemas me ha ayudado a desarrollarlos de manera mucho más clara, a estructurarlos en el mapa en organizadores y derivados, viendo a cuáles les debo dedicar más atención. Incluir los problemas junto a los contenidos que voy a trabajar ha supuesto entender mejor la utilidad y la importancia de dichos contenidos, algo que le he trasmitido al alumnado. Nunca había representado esquemáticamente los contenidos, ni tampoco los había llegado a relacionar unos con otros. Esto me ha permitido disponer de una imagen mental a la que recurrir en la clase para no olvidar la relación entre los mismos, haciéndoles ver a los alumnos la cohesión de las diferentes cuestiones que se plantean en la materia. De la misma manera, he aprendido a equilibrar el tipo de contenidos, dándole especial importancia a los actitudinales.

En cuanto a la metodología, el diseño de un modelo metodológico ha significado para mí una reflexión profunda y constructiva sobre mi manera de dar las clases. Algo que, hasta ahora, no me había replanteado a este nivel. He conseguido dar con un modelo que me ha funcionado y que, con toda seguridad, me va a acompañar en mi docencia futura. El modelo parte de las ideas iniciales de los alumnos, seguido de unas actividades de contraste que finalizan en las ideas finales de los mismos. Las preguntas clave o atractivas han sido fundamentales para el 
desarrollo de este modelo, gracias a ellas he conseguido captar la atención y el interés del alumnado para poder trabajar los contenidos en torno al modelo metodológico previsto. Asimismo, las secuencias de actividades me han hecho posible detallar los procesos de aprendizaje que realizo en clase, tener en cuenta el tiempo y disponer de un esquema que me permita recordar las actividades esenciales para no perderlas de vista.

En cuanto a la evaluación, el cuestionario inicial me ha proporcionado la forma idónea para desarrollar mi docencia advirtiendo el nivel del alumnado. Ahora puedo elaborar un mapa de contenidos y una secuencia de actividades partiendo del nivel de mis alumnos, gracias a la utilización de un cuestionario inicial. Las escaleras de aprendizaje han supuesto para mí toda una revolución evaluadora. Ahora puedo seguir el aprendizaje individual de mis alumnos y, además, saber si mi modelo didáctico funciona o, si por el contrario, necesita de más cambios.

Siempre he repartido a los alumnos una hoja para que evalúen mi enseñanza y es algo que creo que todo docente no debe obviar: la opinión de los alumnos es clave, hay que dialogar e interaccionar también sobre la evaluación para mejorar nuestra manera de enfrentarnos al aprendizaje de los estudiantes. Nunca había llevado a cabo un diario del profesor, algo que, siempre que pueda, voy a incorporar a mi hábito docente, puesto que me ha posibilitado analizar con mucha calma algunas cuestiones ocurridas en clase y que tengo que mejorar como, por ejemplo, no pensar que los temas han quedado resueltos solo porque así lo manifiesten los alumnos más participativos, también están los alumnos que no se expresan en público y hay que preocuparse por saber si ellos comprenden o no lo que se plantea.

Jornadas de Formación e Innovación Docente del Profesorado | № 2 (2019) Esta obra se distribuye con la licencia Creative Commons 


\section{Referencias bibliográficas}

Alba, N. y Porlán, R. (2017). La metodología de enseñanza. En Porlán, R. (Coord.). Enseñanza universitaria. Cómo mejorarla (pp. 37-53). Madrid: Morata.

Bain, K. (2007). Lo que hacen los mejores profesores universitarios. València: Universitat de València.

Claxton, G. (1987). Vivir y aprender. Psicología del desarrollo y del cambio en la vida cotidiana. Madrid: Alianza.

Escribano, A. y del Valle, A. (2008). El aprendizaje basado en problemas. Una propuesta metodológica en Educación Superior. Madrid: Narcea.

Finkel, D. (2008). Dar clases con la boca cerrada. València: Universitat de València.

Porlán, R. (1993). Constructivismo y escuela. Hacia un modelo de enseñanza-aprendizaje basado en la investigación. Sevilla: Díada.

Porlán, R. y Martín, J. (1991): El diario del profesor. Un recurso para la investigación en el aula. Sevilla: Díada.

Porlán, R. y Rivero, A. (1998). El conocimiento de los profesores. Sevilla: Díada.

Porlán, R., Martín del Pozo, R., Rivero, A., Harres, J., Azcárate, P. y Picazzo, M. (2010). El cambio del profesorado de ciencias I: marco teórico y formativo. Enseñanza de las Ciencias, 28 (1), pp. 31-46.

Rivero, A. y Porlán, R. (2017). La evaluación en la enseñanza universitaria. En Porlán, R. (Coord.). Enseñanza universitaria. Cómo mejorarla (pp. 73-91). Madrid: Morata.

Jornadas de Formación e Innovación Docente del Profesorado | № 2 (2019) Esta obra se distribuye con la licencia Creative Commons Reconocimiento-NoComercial-SinObraDerivada 4.0 Internacional (CC BY-NC-ND 4.0.) 